\title{
Comparison of the Exact and Approximate Values of Certain Parameters in Laminar Boundary Layer Flow Using Some Velocity Profiles
}

\author{
Asuquo E. Eyo ${ }^{1}$, Nkem Ogbonna $^{2}$ \& Moses E. Ekpenyong ${ }^{3}$ \\ ${ }^{1}$ Department of Mathematics and Statistics, University of Uyo, Uyo, Nigeria \\ 2 Department of Mathematics, Michael Okpara University of Agriculture, Umudike, Nigeria \\ ${ }^{3}$ Department of Computer Science, University of Uyo, Uyo, Nigeria \\ Correspondence: Asuquo E. Eyo, Department of Mathematics and Statistics, University of Uyo, Uyo, Nigeria. \\ E-mail: asuquoessieneyo@yahoo.com
}

Received: May 24, 2012 Accepted: June 7, 2012 Online Published: September 11, 2012

doi:10.5539/jmr.v4n5p17 URL: http://dx.doi.org/10.5539/jmr.v4n5p17

\begin{abstract}
We apply second, third, fourth, fifth and sixth order velocity profiles to discuss laminar boundary layer flow over a flat plate. Inclusion of these velocity profiles in Von Karman-Pohlhausen (1921) momentum integral equation enables us to determine the approximate values of the parameters namely, (i) boundary layer thickness, (ii) displacement thickness, (iii) momentum thickness, (iv) thickness ratio, (v) skin friction coefficient, (vi) drag coefficient and (vii) the shear rate relation on the plate. Comparison of the approximate values with the exact Blasius (1908) values leads to the determination of the percentage error for each of the above parameters for the different velocity profiles. From the sixth order velocity profile we can predict that higher order velocity profiles will yield greater percentage errors and hence worse and worse results for these parameters except displacement thickness.
\end{abstract}

Keywords: exact values, approximate values, laminar, boundary layer flow, parameters in the flow, velocity profiles

\section{Introduction}

Boundary layer is formed whenever there is relative fluid motion between the solid boundary and the fluid. The velocity within the boundary layer increases from zero at the boundary surface to the velocity of the main stream asymptotically. Therefore the thickness of the boundary layer is arbitrary defined as that distance from the boundary in which the velocity reaches 99 percent of the velocity of the free stream, Schlichting (1968). Boundary layer flow has been a topic of intensive research by various researchers since the development of the concept by Prandtl (1925).

Craft and Lowell (2009) investigated two aspects of oceanic hydrothermal heat flux that are not well understood namely, the relative partitioning of heat flux between high-temperature and low-temperature flows at oceanic spreading ridges and next the hydrothermal behaviour of the near-axis region, where seismic data suggest that a zone of partial melt extends quasi-vertically into the low crust at the East Pacific Rise. They applied steady state boundary layer theory to each system by assuming circulation occurs near a hot isothermal wall that laterally transfers heat to and induces convection within an adjacent fluid-filled medium. In their analysis, they showed that, for the near-axis model, heat transfer in the hydrothermal boundary layer is greater than the input from steady state generation of the oceanic crust by seaflow spreading.

Dorfman (2011) presented a review of universal functions widely used in different areas of boundary layer theory for many years up to the present. Thus, he considered different kinds of universal functions, from simple equations of dimensionless numbers in similarity theory to universal parametric boundary layer equations. Finally, he adopted various universal solutions from almost 100 articles published since the famous Howarth study of Blasius series in 1935 to show the breadth of universal aproaches with application in laminar, turbulent and transition boundary layers in solving non-isothermal and conjugate heat transfer problems as well as in planetary boundary layer problems in meteorology. 
Habib et al. (2009) carried out transient calculation of the boundary layer flow over spills. They presented results of numerical simulations and experimental investigations of the mass flow from evaporating liquid pools. They validated their results of the simulation against experimental data from the open air experiments and also made the comparison of the simulation results with empirical prediction models.

Kim and Changhoon (2009) studied large-eddy simulation of urban boundary layer flow over complex topologies. They used wind tunnel experiment and large eddy simulation to investigate (i) boundary layer flow over arrays of regularly distributed obstacles and (ii) scaled real urban area in which various wind directions were considered. They found that the parameters for the arrays, composed of slender rectangular cylinders whose characteristics were similar to a real urban area rather than cube arrays, were highly sensitive to the wind direction. They also observed that velocity profile along the street canyon with which a wind direction aligned was close to a linear profile rather than a constant or exponential distribution.

Mahmoudian and Scales (2012) investigated irregularity generation associated with dust cloud expansion through a background plasma along a magnetic field. Because of the dust charging process, a boundary layer was produced. Theoretical and computational models were used to study the evolution of relevant plasma instabilities thought to play a dominant role in irregularity production. The relevance of these results to past experimental observations in space and the laboratory for applications to the expansion of naturally or artificially created dust clouds was discussed.

Swan (2012) discussed the universal velocity profile which provides a description of the mean velocity within a turbulent boundary layer. Using dimensional analysis, he suggested that immediately above the viscous sublayer the velocity within the so-called inner region (or wall layer) is given by

$$
\frac{u}{u_{\tau}}=f\left(\frac{y u_{\tau}}{v}\right)
$$

where $u_{\tau}$ is the friction velocity or shear velocity defined by $u_{\tau}=\left(\frac{\tau}{\rho}\right)^{\frac{1}{2}}$ where $\tau$ is the shear stress, $\rho$ the fluid density and $v$ the fluid kinematic viscosity. Based on this assumption, and using several different approaches (momentum transport theory, similarity theory, and dimensional analysis), he proposed that the appropriate form of the function $\mathrm{f}$ is logarithmic and hence

$$
\frac{u}{u_{\tau}}=A \log _{10}\left(\frac{y u_{\tau}}{v}\right)+B
$$

where $\mathrm{A}$ and $\mathrm{B}$ are constants which are typically given values of 5.75 and 5.5 respectively.

Wu and Christensen (2010) discussed spatial structure of a turbulent boundary layer with irregular surface roughness. In doing this they performed particle image velocimetry experiments to study the impact of realistic roughness on the spatial structure of wall turbulence at moderate Reynolds number. The spatial structure of flow over this rough surface near the outer edge of the roghness sublayer was contrasted with that of smooth-wall flow to identify any structural modifications due to roughness. In their analysis they observed hairpin vortex packets in the outer layer of the rough-wall and these were found to contribute heavily to the Reynolds shear stress, consistent with smooth-wall flow. While similar qualitative consistency was observed in comparisons of smooth-and rough-wall two-point correlations,some quantitative differences were also apparent.

Other contributors in the field of boundary layer flow include, notably, Schlichting (1968), Olsson and Turkdogan (1966), Noor Afzal (1983), Huguera (1994), Bohr et al. (1993), etc.

Our objective in this work is to compare the exact and approximate values of certain parameters in laminar boundary layer flow by use of some velocity profiles and show how these profiles vary with the percentage errors of these parameters. In doing this, we employ Karman-Pohlhausen momentum integral equation and the analysis reveals that higher order velocity profiles yield worse result.

\section{Blasius Solution of Two Dimensional Laminar Boundary Layer Equations}

Let $x, y$ be the horizontal and vertical rectangular coordinates respectively and $u, v$ the corresponding velocity components, then the equations for laminar flow are

$$
\begin{gathered}
\frac{\partial u}{\partial x}+\frac{\partial v}{\partial y}=0 \\
u \frac{\partial u}{\partial x}+v \frac{\partial u}{\partial y}=v \frac{\partial^{2} u}{\partial y^{2}}
\end{gathered}
$$




$$
\left.\begin{array}{l}
u=v=0 \text { at } y=0 \\
\frac{\partial u}{\partial y}=0 \text { at } y=h(x) \\
u \rightarrow U \text { as } y \rightarrow \infty
\end{array}\right\}
$$

where $\mathrm{U}$ is the free stream velocity and $\mathrm{u}$ is the velocity at a distance $\mathrm{y}$ above the plate.

A solution of the equation can be found (Blasius, 1908) based on Blasius type of velocity profile given by

$$
u=U f_{1}^{\prime}(\eta), \quad \eta=\left(\frac{U}{v x}\right)^{\frac{1}{2}} y
$$

The equation

$$
f_{1} f_{1}^{\prime \prime}+2 f_{1}^{\prime \prime \prime}=0 \quad(0<\eta<\infty)
$$

is the Blasius equation with boundary conditions

$$
\left.\begin{array}{l}
f_{1}=f_{1}^{\prime}=0 \text { at } \eta=0 \\
f_{1}^{\prime}=1 \text { at } \eta=\infty
\end{array}\right\}
$$

[Here, denotes differentiation with respect to $\eta]$.

Thus the velocity distribution has the Blasius flat-plate profile, and the boundary layer thickness $\delta$ is $0\left(\frac{v x}{U}\right)$. The exact solution of the boundary layer equations (1) - (4) was found by Blasius (1908) through numerical method. Thus for laminar boundary layer flow on a flat plate at a point $\mathrm{x}$ from the leading edge of the plate, Blasius obtained the following results:

(i) Shear rate relation on the plate $f_{1}^{\prime \prime}(0)$

$$
\left[\frac{v x}{U_{0}}\right]^{\frac{1}{2}} \frac{1}{U_{0}}\left[\frac{\partial u}{\partial y}\right]_{y=0}=f_{1}^{\prime \prime}(0)=0.332
$$

(ii) The boundary layer thickness $\delta$ is

$$
\frac{\delta}{x}=\frac{5}{\sqrt{\operatorname{Rex}_{x}}}
$$

(iii) The displacement thickness $\delta_{1}$ is

$$
\frac{\delta_{1}}{x}=\frac{1.7208}{\sqrt{\operatorname{Re}_{x}}}
$$

(iv) The momentum thickness $\delta_{2}$ is

$$
\frac{\delta_{2}}{x}=\frac{0.664}{\sqrt{R e_{x}}}
$$

so that (v) The thickness ratio $H^{*}$ is

$$
H^{*}=\frac{\delta_{1}}{\delta_{2}}=2.5915
$$

(vi) Drag coefficient for the plate over one surface $C_{D}$ is

$$
C_{D}=\frac{1.328}{\sqrt{R e_{x}}}
$$

(vii) Skin friction coefficient $C_{f}$ is

$$
C_{f}=\frac{0.664}{\sqrt{R e_{x}}}
$$

\section{Second Order Velocity Profile}

We take the velocity profile in the form

$$
u=U(x) f(\eta), \quad \eta=\frac{y}{\delta(x)}
$$

where

$$
f(\eta)=2 \eta-\eta^{2}
$$


and such that $f(\eta)$ satisfies the following conditions:

$$
\left.\begin{array}{ll}
f(\eta)=0, & \eta=0 \\
f(\eta)=1, & \eta=1 \\
f^{\prime}(\eta)=0, & \eta=1
\end{array}\right\}
$$

\section{Karman-Pohlhausen Momentum Integral Equation}

This integral equation for two dimensional laminar flow (Pohlhausen, 1921) is given by

$$
\frac{d}{d x} \int_{0}^{\delta}\left(U u-u^{2}\right) d y=v\left(\frac{\partial u}{\partial y}\right)_{y=0}
$$

From (15) and (16) we find

$$
u=U\left(2 \eta-\eta^{2}\right), \quad \eta=\frac{y}{\delta}
$$

so that

$$
\left(\frac{\partial u}{\partial y}\right)_{y=0}=\left.\frac{\partial u}{\partial \eta} \frac{\partial \eta}{\partial y}\right|_{\eta=0}=\frac{2 U}{\delta}
$$

Hence, the shear stress on the plate becomes

$$
\tau_{0}=\mu\left(\frac{\partial u}{\partial y}\right)_{y=0}=\frac{2 \mu U}{\delta}
$$

Shear Rate Relation on the Plate $f^{\prime \prime}(0)$

Now

$$
\begin{aligned}
& \int_{0}^{\delta} U u d y=U^{2} \delta \int_{0}^{1}\left(2 \eta-\eta^{2}\right) d \eta=U^{2} \delta \cdot \frac{2}{3} \\
& \int_{0}^{\delta} u^{2} d y=U^{2} \delta \int_{0}^{1}\left(2 \eta-\eta^{2}\right)^{2} d \eta=U^{2} \delta \cdot \frac{8}{15}
\end{aligned}
$$

Substituting (21), (22) and (23) in (18) we have

$$
\frac{2}{15} U^{2} \frac{d}{d x} \delta=v U \cdot \frac{2}{\delta}
$$

giving on integration

$$
\delta^{2}=30 \frac{v x}{U}+C
$$

where $\mathrm{C}$ is a constant of integration. If (25) were to remain valid as $x \rightarrow 0$, then $C=0$. Consequently,

$$
\delta^{2}=30 \frac{v x}{U}
$$

or

$$
\delta=\left[\frac{v x^{\frac{1}{2}}}{U}\right]^{\frac{1}{2}} \times(30)^{\frac{1}{2}}
$$

Hence

$$
\frac{d}{d x} \delta=\frac{1}{2}\left[\frac{v x}{U}\right]^{\frac{1}{2}} \frac{v}{U} \times(30)^{\frac{1}{2}}
$$

Comparing (28) with (24) we get

$$
v U \cdot \frac{2}{\delta} \cdot \frac{1}{U^{2}}\left[\frac{15}{2}\right]=\frac{1}{2}\left[\frac{v x}{U}\right]^{-\frac{1}{2}} \frac{v}{U} \times(30)^{\frac{1}{2}}
$$

i.e.

$$
\left[\frac{v x}{U}\right]^{\frac{1}{2}} \frac{1}{U}\left[\frac{\partial u}{\partial y}\right]_{y=0}=f^{\prime \prime}(0)=\frac{1}{2}\left[\frac{2}{15}\right] \times(30)^{\frac{1}{2}}
$$


or

$$
f^{\prime \prime}(0)=0.36514
$$

which is the required shear rate relation on the plate.

\section{Boundary Layer Thickness $\delta$}

Using (27), we find

$$
\delta=\left[\frac{v x}{U}\right]^{\frac{1}{2}} \times 5.477225
$$

which can be expressed as

$$
\delta=\frac{x}{\left[\frac{U x}{v}\right]^{\frac{1}{2}}} \times 5.477225
$$

or

$$
\frac{\delta}{x}=\frac{5.477225}{\sqrt{R e_{x}}}
$$

\section{Displacement Thickness $\delta_{1}$}

From Karman-Pohlhausen (1921),

$$
\delta_{1}=\int_{0}^{\delta}\left(1-\frac{u}{U}\right) d y
$$

Again, substituting (15) and (16) into (34) we find

$$
\delta_{1}=\delta \int_{0}^{1}\left[1-\left(2 \eta-\eta^{2}\right)\right] d \eta=\frac{1}{3} \delta
$$

Applying (33) in (35), we obtain

$$
\frac{\delta_{1}}{x}=\frac{1.82574}{\sqrt{R e_{x}}}
$$

Momentum Thickness $\delta_{2}$

Again, from Karman-Pohlansen (1921),

$$
\delta_{2}=\int_{0}^{\delta} \frac{u}{U}\left(1-\frac{u}{U}\right) d y
$$

Substituting (15) and (16) into (37) gives

$$
\delta_{2}=\delta \int_{0}^{1}\left(2 \eta-\eta^{2}\right)\left[1-\left(2 \eta-\eta^{2}\right)\right] d \eta
$$

which on integration and simplification yields

$$
\delta_{2}=\frac{2}{15} \delta
$$

Using (33) in (38) leads to

$$
\frac{\delta_{2}}{x}=\frac{0.73029}{\sqrt{\operatorname{Re}_{x}}}
$$

Thickness Ratio (or Shape Factor) $H^{*}$

Here, using (36) and (39) we find

$$
H^{*}=\frac{\delta_{1}}{\delta_{2}}=2.50
$$

\section{Skin Friction Coefficient $C_{f}$}

Substituting (33) into (21), the shear stress on the plate $\tau_{0}$ becomes

$$
\tau_{0}=\frac{(2 \mu U)}{\left(\frac{5.477225 \times x}{\sqrt{R e_{x}}}\right)}
$$


or

$$
\tau_{0}=\frac{2 \mu U}{5.477225} \times \frac{\sqrt{\operatorname{Re}_{x}}}{x}
$$

But from Schlichting (1968), skin friction coefficient is given by

$$
C_{f}=\frac{\tau_{0}}{\frac{1}{2} \rho U^{2}}
$$

so that substituting (42) in (43) we find

$$
C_{f}=\frac{0.73029}{\sqrt{\operatorname{Re}_{x}}}
$$

\section{Drag Coefficient $C_{D}$}

The drag force $F_{D}$ on one side of the plate of width B and length L (Schlichting, 1968), is

$$
F_{D}=\int_{0}^{L} \tau_{0} \times B d x
$$

Substituting (42) into (45) we have

$$
F_{D}=\frac{2 \mu U B}{5.477225} \int_{0}^{L} \frac{\sqrt{R e_{x}}}{x} d x
$$

i.e.

$$
F_{D}=\frac{2 \mu U B}{5.477225} \int_{0}^{L} \sqrt{\frac{\rho U x}{\mu}} \cdot \frac{1}{x} d x
$$

or

$$
F_{D}=\frac{2 \sqrt{\mu} U B \sqrt{\rho U}}{5.477225} \int_{0}^{L} x^{-\frac{1}{2}} d x
$$

After integration and simplification (48) gives

$$
F_{D}=\frac{4 \sqrt{\mu} U B \sqrt{\rho U L}}{5.477225}
$$

The drag coefficient becomes (Schlichting, 1968)

$$
C_{D}=\frac{F_{D}}{\frac{1}{2} \rho A U^{2}}=\frac{F_{D}}{\frac{1}{2} \rho U^{2} \times B \times L} \quad(\text { where } A=B \times L)
$$

so that using (49) in (50) and simplifying we obtain

$$
C_{D}=\frac{8}{5.477225} \times \frac{\sqrt{\mu}}{\sqrt{\rho u L}}
$$

or

$$
C_{D}=\frac{1.460593}{\sqrt{\operatorname{Re}_{L}}}
$$

\section{Third Order Velocity Profile}

The form of the velocity profile here is

$$
u=U(x) f(\eta)
$$

where

$$
f(\eta)=\frac{3}{2} \eta-\frac{1}{2} \eta^{3}
$$

i.e

$$
u=U\left(\frac{3}{2} \eta-\frac{1}{2} \eta^{3}\right), \quad \eta=\frac{y}{\delta}
$$

so that

$$
\left(\frac{\partial u}{\partial y}\right)_{y=0}=\frac{3 U}{2 \delta}
$$


Hence

$$
\tau_{0}=\frac{3 \mu U}{2 \delta}
$$

Shear Rate Relation on the Plate $f^{\prime \prime}(0)$

Now

$$
\begin{aligned}
& \int_{0}^{\delta} U u d y=U^{2} \delta \int_{0}^{1}\left(\frac{3}{2} \eta-\frac{1}{2} \eta^{3}\right) d \eta=U^{2} \delta \cdot \frac{5}{8} \\
& \int_{0}^{\delta} u^{2} d y=U^{2} \delta \int_{0}^{1}\left(\frac{3}{2} \eta-\frac{1}{2} \eta^{3}\right)^{2} d \eta=U^{2} \delta \cdot \frac{17}{35}
\end{aligned}
$$

Substituting (56), (58) and (59) iinto (18) we find

$$
\frac{39}{280} U^{2} \frac{d}{d x} \delta=v U \cdot \frac{3}{2 \delta}
$$

which leads to

$$
\delta^{2}=\frac{840}{39} \cdot \frac{v x}{U}
$$

as in (26).

Thus

$$
\delta=\left[\frac{840}{39}\right]^{\frac{1}{2}} \cdot\left[\frac{v x}{U}\right]^{\frac{1}{2}}
$$

and

$$
\frac{d}{d x} \delta=\frac{1}{2}\left[\frac{840}{39}\right]^{\frac{1}{2}} \frac{v}{U}\left[\frac{v x}{U}\right]^{-\frac{1}{2}}
$$

Comparing (63) and (60) we have

$$
v U \cdot \frac{3}{2 \delta} \cdot \frac{1}{U^{2}}\left[\frac{280}{39}\right]=\frac{1}{2}\left[\frac{840}{39}\right]^{\frac{1}{2}} \frac{v}{U}\left[\frac{v x}{U}\right]^{-\frac{1}{2}}
$$

resulting in

$$
\left[\frac{v x}{U}\right]^{\frac{1}{2}} \frac{1}{U}\left[\frac{\partial u}{\partial y}\right]_{y=0}=f^{\prime \prime}(0)=\frac{1}{2}\left[\frac{840}{39}\right]^{\frac{1}{2}}\left[\frac{39}{280}\right]
$$

Thus

$$
f^{\prime \prime}(0)=0.323209
$$

\section{Boundary Layer Thickness $\delta$}

From (62),

$$
\delta=\left[\frac{v x}{U}\right]^{\frac{1}{2}} \times 4.6409548
$$

or

$$
\frac{\delta}{x}=\frac{4.6409548}{\sqrt{R e_{x}}}
$$

\section{Dispacement Thickness $\delta_{1}$}

Substituting (53) and (54) into (34) we obtain

$$
\delta_{1}=\delta \int_{0}^{1}\left[1-\left(\frac{3}{2} \eta-\frac{1}{2} \eta^{3}\right)\right] d \eta=\frac{3}{8} \delta
$$

which on using (67) in (68) yields

$$
\frac{\delta_{1}}{x}=\frac{1.740358}{\sqrt{\operatorname{Re} x}}
$$

Momentum Thickness $\delta_{2}$ 
Using (53) and (54) in (37) gives

$$
\delta_{2}=\delta \int_{0}^{1}\left(\frac{3}{2} \eta-\frac{1}{2} \eta^{3}\right)\left[1-\left(\frac{3}{2} \eta-\frac{1}{2} \eta^{3}\right)\right] d \eta=\frac{39}{280} \delta
$$

Applying (67) in (70) we find

$$
\frac{\delta_{2}}{x}=\frac{0.6464187}{\sqrt{\operatorname{Re}_{x}}}
$$

\section{Thickness Ratio $H^{*}$}

Using (68) and (70) we have

$$
H^{*}=2.69230769
$$

Skin Friction Coefficient $C_{f}$

Substituting (67) into (57) leads to

$$
\frac{\left(\frac{3 \mu U}{2}\right)}{\left(\frac{4.6409548}{\sqrt{\operatorname{Re}_{x}}} \times x\right)}
$$

i.e

$$
\tau_{0}=\frac{3 \mu U}{9.2819096} \times \frac{\sqrt{R e_{x}}}{x}
$$

Further substitution of (74) into (43) yields

$$
C_{f}=\frac{0.6464187}{\sqrt{R e_{x}}}
$$

\section{Drag Coefficient $C_{D}$}

Substituting (74) into (45) we find

$$
F_{D}=\frac{3 \mu U B}{9.2819096} \int_{0}^{L} \frac{\sqrt{\operatorname{Re}_{x}}}{x} d x
$$

i.e

$$
F_{D}=\frac{3 \sqrt{\mu} U B \sqrt{\rho U}}{9.2819096} \int_{0}^{L} x^{-\frac{1}{2}} d x
$$

or

$$
F_{D}=\frac{6 \sqrt{\mu} U B \sqrt{\rho U L}}{9.2819096}
$$

Substituting (78) in (50) we have after simplification

$$
C_{D}=\frac{12}{9.2819096} \times \frac{\sqrt{\mu}}{\sqrt{\mu U L}}
$$

whence

$$
C_{D}=\frac{1.2928374}{\sqrt{\operatorname{Re}_{L}}}
$$

\section{Fourth Order Velocity Profile}

Here the profile is taken as

$$
u=U(x) f(\eta)
$$

with

$$
f(\eta)=2 \eta-2 \eta^{3}+\eta^{4}
$$

or

$$
u=U\left(2 \eta-2 \eta^{3}+\eta^{4}\right)
$$

Now

$$
\left(\frac{\partial u}{\partial y}\right)_{y=0}=\frac{2 U}{\delta}
$$


so that

$$
\tau_{0}=\frac{2 \mu U}{\delta}
$$

Shear Rate Relation on the Plate $f^{\prime \prime}(0)$

$$
\begin{aligned}
& \int_{0}^{\delta} U u d y=U^{2} \delta \int_{0}^{1}\left(2 \eta-2 \eta^{3}+\eta^{4}\right) d \eta=U^{2} \delta \frac{7}{10} \\
& \int_{\delta}^{\delta} u^{2} d y=U^{2} \delta \int_{0}^{1}\left(2 \eta-2 \eta^{3}+\eta^{4}\right)^{2} d \eta=U^{2} \delta \frac{367}{630}
\end{aligned}
$$

Using (84), (86) and (87) in (18) we have

$$
\frac{37}{315} U^{2} \frac{d}{d x} \delta=v U \frac{2}{\delta}
$$

which on integration gives

$$
\delta^{2}=\frac{1260}{37} \cdot \frac{v x}{U}
$$

Hence

$$
\delta=\left[\frac{1260}{37}\right]^{\frac{1}{2}}\left[\frac{v x}{U}\right]^{\frac{1}{2}}
$$

and

$$
\frac{d}{d x} \delta=\frac{1}{2}\left[\frac{1260}{37}\right]^{\frac{1}{2}} \frac{v}{U}\left[\frac{v x}{U}\right]^{-\frac{1}{2}}
$$

Comparing (91) and (88) we find

$$
v U \cdot \frac{2}{\delta} \cdot \frac{1}{U^{2}}\left[\frac{315}{37}\right]=\frac{1}{2}\left[\frac{1260}{37}\right]^{\frac{1}{2}} \frac{v}{U}\left[\frac{v x}{U}\right]^{-\frac{1}{2}}
$$

yielding

$$
\left[\frac{v x}{U}\right]^{\frac{1}{2}} \frac{1}{U}\left[\frac{\partial u}{\partial y}\right]_{y=0}=f^{\prime \prime}(0)=\frac{1}{2}\left[\frac{1260}{37}\right]^{\frac{1}{2}}\left[\frac{37}{315}\right]
$$

Thus

$$
f^{\prime \prime}(0)=0.34272484
$$

\section{Boundary Layer Thickness $\delta$}

From (90),

$$
\delta=\left[\frac{v x}{U}\right]^{\frac{1}{2}} \times 5.835585
$$

whence

$$
\frac{\delta}{x}=\frac{5.835585}{\sqrt{R e_{x}}}
$$

\section{Displacement Thicknessn $\delta_{1}$}

Sustituting (81) and (82) into (34) we find

$$
\delta_{1}=\delta \int_{0}^{1}\left[1-\left(2 \eta-2 \eta^{3}+\eta^{4}\right)\right] d \eta=\frac{3}{10} \delta
$$

Using (95) in (96), we obtain

$$
\frac{\delta_{1}}{x}=\frac{1.7506755}{\sqrt{\operatorname{Re}_{x}}}
$$

Momentum Thickness $\delta_{2}$

Substituting (81) and (82) into (37) gives

$$
\delta_{2}=\delta \int_{0}^{1}\left(2 \eta-2 \eta^{3}+\eta^{4}\right)\left[1-\left(2 \eta-2 \eta^{3}+\eta^{4}\right)\right] d \eta=\frac{37}{315} \delta
$$


Applying (95) in (98) results in

$$
\frac{\delta_{2}}{x}=\frac{0.685449666}{\sqrt{R e_{x}}}
$$

\section{Thickness Ratio $H^{*}$}

From (96) and (98) we get

$$
H^{*}=2.554054
$$

\section{Skin Friction Coefficient}

Substituting (95) into (85) we find

$$
\tau_{0}=\frac{(2 \mu U)}{\left(\frac{5.835585}{\sqrt{R e_{x}}} \times x\right)}
$$

or

$$
\tau_{0}=\frac{2 \mu U}{5.835585} \times \frac{\sqrt{R e_{x}}}{x}
$$

Using (102) in (43) gives

$$
C_{f}=\frac{0.6854497}{\sqrt{R e_{x}}}
$$

Drag Coefficient $C_{D}$

Substituting (102) into (45) we get

$$
F_{D}=\frac{2 \mu U B}{5.835585} \int_{0}^{L} \frac{\sqrt{R e_{x}}}{x} d x
$$

which on integration gives

$$
F_{D}=\frac{4 \sqrt{\mu} U B \sqrt{\rho U L}}{5.835585}
$$

Using (105) in (50) we obtain after simplification

$$
C_{D}=\frac{8}{5.835585} \times \frac{\sqrt{\mu}}{\sqrt{\rho U L}}
$$

or

$$
C_{D}=\frac{1.3708994}{\sqrt{R e_{x}}}
$$

\section{Application of Fifth and Sixth Order Velocity Profiles}

Similar analyis using fifth and sixth order velocity profiles yields the following results:

\section{Fifth Order Velocity Profile}

The form of this profile is

$$
u=U(x) f(\eta)
$$

with

$$
f(\eta)=\frac{5}{3} \eta-\frac{5}{3} \eta^{4}+\eta^{5}, \quad \eta=\frac{y}{\delta}
$$

or

$$
u=U\left(\frac{5}{3} \eta-\frac{5}{3} \eta^{4}+\eta^{5}\right)
$$

Result:

Boundary layer thickness $\delta$

$$
\frac{\delta}{x}=\frac{5.17936}{\sqrt{R e_{x}}}
$$

Dispacement thickness $\delta_{1}$

$$
\frac{\delta_{1}}{x}=\frac{1.72645}{\sqrt{R e_{x}}}
$$


Momentum thickness $\delta_{2}$

$$
\frac{\delta_{2}}{x}=\frac{0.64357}{\sqrt{R e_{x}}}
$$

Thickness ratio $H^{*}$

$$
H^{*}=2.68258
$$

Skin friction coefficient $C_{f}$

$$
C_{f}=\frac{0.64357}{\sqrt{R e_{x}}}
$$

Drag coefficient $C_{D}$

$$
C_{D}=\frac{1.28715}{\sqrt{R e_{L}}}
$$

Shear Rate Relation on the Plate $f^{\prime \prime}(0)$

$$
f^{\prime \prime}(0)=0.32178
$$

\section{Sixth Order Velocity Profile}

The profile is taken as

$$
u=U(x) f(\eta)
$$

where

$$
f(\eta)=2 \eta-2 \eta^{4}+\eta^{6}
$$

or

$$
u=U\left(2 \eta-2 \eta^{4}+\eta^{6}\right)
$$

Result:

Boundary layer thickness $\delta$

$$
\frac{\delta}{x}=\frac{6.85486}{\sqrt{\operatorname{Re}_{x}}}
$$

Displacement thickness $\delta_{1}$

$$
\frac{\delta_{1}}{x}=\frac{1.76267}{\sqrt{R e_{x}}}
$$

Momentum thickness $\delta_{2}$

$$
\frac{\delta_{2}}{x}=\frac{0.58352}{\sqrt{R e_{x}}}
$$

Thickness ratio $H^{*}$

$$
H^{*}=3.02073
$$

Skin friction coefficient $C_{f}$

$$
C_{f}=\frac{0.58352}{\sqrt{R e_{x}}}
$$

Drag coefficient $C_{D}$

$$
C_{D}=\frac{1.16705}{\sqrt{R e_{L}}}
$$

Shear rate relation on the plate $f^{\prime \prime}(0)$

$$
f^{\prime \prime}(0)=0.29176
$$

\section{Final Result}

The final result for the parameters $\delta, \delta_{1}, \delta_{2}, H^{*}, C_{f}, C_{D}$ and $f^{\prime \prime}(0)$ with respect to the second, third, fourth, fifth and sixth order velocity profiles together with the exact Blasius solution is shown in Table 1, while the percentage error of these parameters is displayed in Table 2. 
Table 1. Approximate values of the parameters with exact values shown at the bottom of the table

\begin{tabular}{lccccccc}
\hline Velocity profile & $\frac{\delta}{x}$ & $\frac{\delta_{1}}{x}$ & $\frac{\delta_{2}}{x}$ & $H^{*}=\frac{\delta_{1}}{\delta_{2}}$ & $C_{f}$ & $C_{D}$ & $f^{\prime \prime}(0)$ \\
\hline$u=U\left(2 \eta-\eta^{2}\right)$ & $\frac{5.4772}{\sqrt{R e_{x}}}$ & $\frac{1.8257}{\sqrt{R e_{x}}}$ & $\frac{0.7302}{\sqrt{R e_{x}}}$ & 2.50 & $\frac{0.7302}{\sqrt{R e_{x}}}$ & $\frac{1.4605}{\sqrt{R e_{L}}}$ & 0.3651 \\
$u=U\left(\frac{3}{2} \eta-\frac{1}{2} \eta^{3}\right)$ & $\frac{4.6409}{\sqrt{R e_{x}}}$ & $\frac{1.7403}{\sqrt{R e_{x}}}$ & $\frac{0.6464}{\sqrt{R e_{x}}}$ & 2.6923 & $\frac{0.6464}{\sqrt{R e_{x}}}$ & $\frac{1.2928}{\sqrt{R e_{L}}}$ & 0.3232 \\
$u=U\left(2 \eta-2 \eta^{3}+\eta^{4}\right)$ & $\frac{5.8355}{\sqrt{R e_{x}}}$ & $\frac{1.7506}{\sqrt{R e_{x}}}$ & $\frac{0.6854}{\sqrt{R e_{x}}}$ & 2.5540 & $\frac{0.6854}{\sqrt{R e_{x}}}$ & $\frac{1.3708}{\sqrt{R e_{L}}}$ & 0.3427 \\
$u=U\left(\frac{5}{3} \eta-\frac{5}{3} \eta^{4}+\eta^{5}\right)$ & $\frac{5.1793}{\sqrt{R e_{x}}}$ & $\frac{1.7264}{\sqrt{R e_{x}}}$ & $\frac{0.6435}{\sqrt{R e_{x}}}$ & 2.6825 & $\frac{0.6435}{\sqrt{R e_{x}}}$ & $\frac{1.2871}{\sqrt{R e_{L}}}$ & 0.3217 \\
$u=U\left(2 \eta-2 \eta^{4}+\eta^{6}\right)$ & $\frac{6.8548}{\sqrt{R e_{x}}}$ & $\frac{1.7627}{\sqrt{R e_{x}}}$ & $\frac{0.5835}{\sqrt{R e_{x}}}$ & 3.0207 & $\frac{0.5835}{\sqrt{R e_{x}}}$ & $\frac{1.16705}{\sqrt{R e_{L}}}$ & 0.2917 \\
Blasius Exact Solution & $\frac{5}{\sqrt{R e_{x}}}$ & $\frac{1.7208}{\sqrt{R e_{x}}}$ & $\frac{0.664}{\sqrt{R e_{x}}}$ & 2.5915 & $\frac{0.664}{\sqrt{R e_{x}}}$ & $\frac{1.328}{\sqrt{R e_{x}}}$ & 0.332 \\
\hline
\end{tabular}

Table 2. Percentage errors of the parameters

\begin{tabular}{llllllll}
\hline & \multicolumn{7}{c}{ Percentage error of the parameters } \\
Velocity profile & $\frac{\delta}{x}$ & $\frac{\delta_{1}}{x}$ & $\frac{\delta_{2}}{x}$ & $H^{*}=\frac{\delta_{1}}{\delta_{2}}$ & $C_{f}$ & $C_{D}$ & $f^{\prime \prime}(0)$ \\
\hline$u=U\left(2 \eta-\eta^{2}\right)$ & 9.54 & 6.09 & 9.96 & 3.53 & 9.96 & 9.97 & 9.96 \\
$u=U\left(\frac{3}{2} \eta-\frac{1}{2} \eta^{3}\right)$ & 7.18 & 1.13 & 2.65 & 3.88 & 2.65 & 2.65 & 2.65 \\
$u=U\left(2 \eta-2 \eta^{3}+\eta^{4}\right)$ & 16.71 & 1.73 & 3.22 & 1.44 & 3.22 & 3.22 & 3.22 \\
$u=U\left(\frac{5}{3} \eta-\frac{5}{3} \eta^{4}+\eta^{5}\right)$ & 3.58 & 0.32 & 3.08 & 3.51 & 3.08 & 3.07 & 3.10 \\
$u=U\left(2 \eta-2 \eta^{4}+\eta^{6}\right)$ & 37.09 & 2.43 & 12.12 & 16.56 & 12.12 & 12.11 & 12.13 \\
\hline
\end{tabular}

\section{Discussion and Conclusion}

Table 1 shows the approximate values of the parameters for each of the profile together with the exact Blasius solution shown at the bottom of the table, while Table 2 shows the percentage error of these parameters for each profile. In Table 2, it is observed that the percentage error with respect to the boundary layer thickness $\delta$ increases as the even order of the velocity profile increases (or decreases as the odd order of the velocity profile increases). This trend is also illustrated graphically below. Table 2 also reveals that the percentage errors for the displacement thickness $\delta_{1}$ are smaller than those of the momentum thickness $\delta_{2}$. Hence the graph for displacement thickness falls below that of the monentum thickness.

Furthermore, Table 2 shows that the parameters $C_{f}, C_{D}$ and $f^{\prime \prime}(0)$ have very nearly the same percentage error as that of $\delta_{2}$ for each of the velocity profile, and hence their graphs will closely be like that of the momentum thickness $\delta_{2}$. From the result of the sixth order profile we can conclude generally that the higher the order of the velocity profile the worse the results for the parameters. Finally, the velocity profiles considered in this work show that the flow is attached (i.e not separated) as the profiles satisfy the conditions (17) of this work. Also, it should be mentioned that the first order profile is not applicable here because the flow in this case is not attached. 


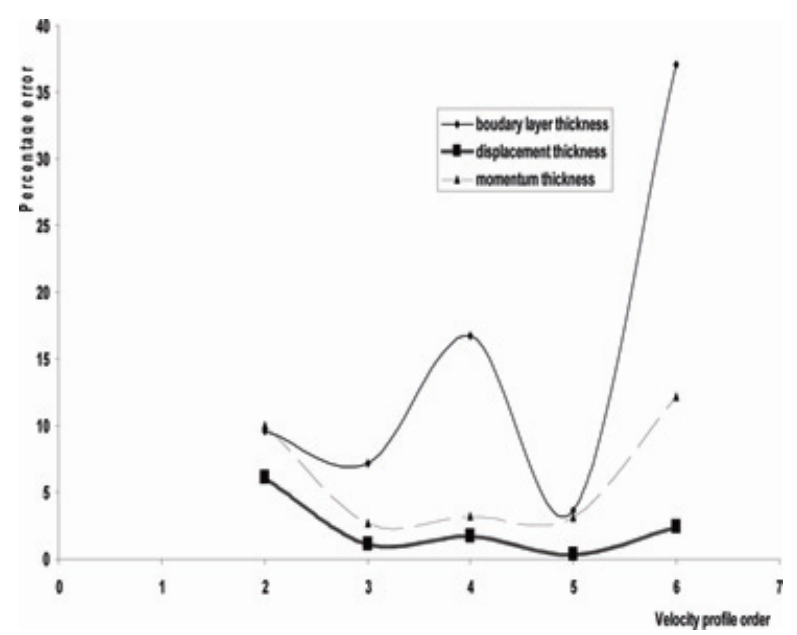

Figure 1. Graph of percentage error versus velocity profile order

\section{References}

Afzal, N. (1983). Analysis of a Turbulent Boundary Layer Subjected to a Strong Adverse Pressure Gradient. International Journal of Engineering and Science, 21(6), 563-576. http://dx.doi.org/10.1016/0020-7225(83)90104-0

Bhor, T., Dimond, P., \& Putkaradize, V. (1993). Shallow Water Approach to Circular Hydraulic Jump. Journal of Fluid Mechanics, 254, 635-645. http://dx.doi.org/10.1017/S0022112093002289

Blasius, H. (1908). Grenzchichten in Flussigluiten mit klener reibung. Zeitschrift fur angewande Mathematik and Physik, 56, English Translation NACA TM. No. 1256.

Craft, K. L., \& Lowell, R. P. (2009). A Boundary Layer Model for Submarine Hydrothermal Heat Flows at on-axis and near-axis Regions. Geochem. Geophys. Geosyst., 10(12), Q12012.

Dorfman, A. (2011). Universal Functions in Boundary Layer Theory. Fundamental Journal of Thermal Science and Engineering, 1(1), 1-72.

Habib, A., Schalau, B., Acikalin, A., \& Steinbach, J. (2009). Transient Calculation of the Boundary Layer Flow over Spills. Chemical Engineering and Technology, 32(2), 306-311. http://dx.doi.org/10.1002/ceat.200800527

Huguera, F. J. (1994). The Hydraulic Jump in a Viscous Laminar Flow. Journal of Fluid Mechanics, 274, 69-92. http://dx.doi.org/10.1017/S0022112094002041

Kim, B., \& Changhoon, L. (2009). Large-Eddy Simulation of Urban Boundary layer Flow over Complex Topologies. American Physical Society, 62nd Annual Meeting of the APS Division of Fluid Dynamics.

Mahmoudian, A., \& Scales, W. A. (2012). Irregularity Excitation Associated with Charged Dust Cloud Boundary Layers. Journal of Geophysical Research, 117, A02304, pp. 11. http://dx.doi.org/10.1029/2011JA017204

Olsson, R. C., \& Turkdogan, E. T. (1966). Radial Spread of a Liquid Stream on a Horizontal Plate. Nature, 211, 813-816. http://dx.doi.org/10.1038/211813a0

Pohlhausen, K. (1921). Zur naherungsweisen Integration der Differentialgleichung der laminarer Grenzshicht. ZAMM., 1, 252-368. http://dx.doi.org/10.1002/zamm.19210010402

Prandtl, L. (1925). Bericht uber Untersuchungen Zur ausgebildeten Turbulenz. Z. angew. Math. Mech., 5(2), p. 136.

Schlichting, H, (1968). Boundary Layer Theory (6th ed.). New York: McGraw-Hill.

Swan, C. (2012). Univesal Velocity Profile. A - to - Z Guide to Thermodynamics, Heat and Mass Transfer, and Fluids Engienering. http://dx.doi.org/10.1615/AtoZ.u.universal_velocity_profile

Wu, Y., \& Christensen, K. (2010). Spatial Structure of a Turbulent Boundary Layer with Irregular Surface Roughness. Journal of Fluid Mechanics, 655, 380 - 418. http://dx.doi.org/10.1017/S0022112010000960 